I. P. Shestakov $\cdot$ E. Zelmanov

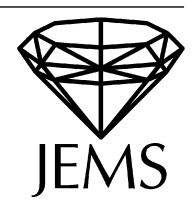

\title{
Some examples of nil Lie algebras
}

Received June 2, 2007

Abstract. Generalizing Petrogradsky's construction, we give examples of infinite-dimensional nil Lie algebras of finite Gelfand-Kirillov dimension over any field of positive characteristic.

The first examples of infinite-dimensional affine nil algebras were constructed by E. S. Golod and I. R. Shafarevich [GS]. These algebras have a strong exponential growth. Later L. Bartholdi and R. I. Grigorchuk [BG] showed that the Lie algebra associated to the "self-similar" Grigorchuk group is graded-nil and has Gelfand-Kirillov dimension 1. Using this result $\mathrm{L}$. Bartholdi $[\overline{\mathrm{B}}]$ was able to construct an infinite-dimensional affine graded-nil associative algebra of Gelfand-Kirillov dimension 2 over a finite field of characteristic 2. Recently T. Lenagan and A. Smoktunowicz [LS] constructed a family of infinite-dimensional affine nil algebras of finite Gelfand-Kirillov dimension over an arbitrary countable field.

In $[\bar{P}]$ V. Petrogradsky found an infinite-dimensional two-generated "self-similar" Lie algebra $L$ over an arbitrary field of characteristic 2 such that (i) $L$ is nil, (ii) $1<$ $\operatorname{GKdim} L<2$.

In this paper we generalize Petrogradsky's construction and extend it to algebras over fields of arbitrary positive characteristic.

Let $p$ be a prime number; $F$ a field of characteristc $p ; \widehat{T}=F\left[t_{0}, t_{1}, \ldots\right]$ the algebra of truncated polynomials in countably many variables $t_{0}, t_{1}, \ldots ; t_{i}^{p}=0, i \geq 0$. Let $T$ be the subalgebra of $\widehat{T}$ consisting of polynomials with zero constant term. Write $\widehat{T}(k)=$ $F\left[t_{0}, t_{1}, \ldots, t_{k}\right]$ (for $k<0$ we let $\widehat{T}(k)=F \cdot 1$ ) and consider the following Lie algebra of derivations of $\widehat{T}$ :

$$
\mathcal{D}=\left\{\sum_{i=1}^{\infty} a_{i} \partial_{i} \mid a_{i} \in \widehat{T}(i-2)\right\}
$$

where $\partial_{i}=d / d t_{i}$. For $k \geq 1$, let

$$
M_{k}=\mathcal{D} \cap\left\{\sum_{i \leq k-1} \widehat{T} \partial_{i}+\sum_{j=0}^{\infty} T^{1+j(p-1)} \partial_{k+j}\right\} .
$$

I. P. Shestakov: Instituto de Matemática e Estatística, Universidade de São Paulo, Caixa Postal 66281, CEP 05315-970, São Paulo, Brasil; e-mail: shestak@ ime.usp.br

E. Zelmanov: Department of Mathematics, UCSD, La Jolla, CA 92093-0112, USA;

e-mail: ezelmano@math.ucsd.edu 
In other words, a derivation $\sum_{i=1}^{\infty} a_{i} \partial_{i}$ lies in $M_{k}$ if $a_{i} \in \widehat{T}(i-2) \cap T^{1+(i-k)(p-1)}$ for $i \geq k$. Clearly, $M_{1} \subset M_{2} \subset \cdots$.

Lemma 1. (a) $\left[M_{k}, M_{q}\right] \subseteq M_{\max (k, q)+1}$, (b) $a^{p} \in M_{k+1}$ for all $a \in M_{k}$.

Proof. (a) is obvious. Let us check (b). Let $d=\sum_{i=1}^{\infty} a_{i} \partial_{i} \in M_{k}$. Since $a_{i} \in \widehat{T}(i-2)$ it follows that $\left(a_{i} \partial_{i}\right)^{p}=a_{i}^{p} \partial_{i}^{p}=0$. Now consider a commutator $\left[a_{i_{1}} \partial_{i_{1}}, \ldots, a_{i_{p}} \partial_{i_{p}}\right]=$ $b \partial_{i_{s}}$, where $b \in \widehat{T}$ and $i_{s}=\max \left\{i_{1}, \ldots, i_{p}\right\}$.

The coefficient $b$ is an expression in (repeated) $\left(\partial_{i_{1}}, \ldots, \partial_{i_{s-1}}, \partial_{i_{s+1}}, \ldots, \partial_{i_{p}}\right)$-derivations of $a_{i_{1}}, \ldots, a_{i_{p}}$. Let $i_{s} \geq k+1$. Then $a_{i_{s}} \in T^{1+\left(i_{s}-k\right)(p-1)}$, no more than $p-1$ derivations could have been applied to $a_{i_{s}}$, hence the derivative lies in $T^{1+\left(i_{s}-k\right)(p-1)-(p-1)}=$ $T^{1+\left(i_{s}-k-1\right)(p-1)}$.

Let $M=\bigcup_{k \geq 1} M_{k}$. From Lemma 1 it follows that $M$ is a Lie subalgebra of $\mathcal{D}$, which is closed with respect to $p$-th powers. Let

$$
\mathcal{D}_{2}=\left\{\sum_{i=1}^{\infty} a_{i} \partial_{i} \mid a_{i} \in T^{2} \cap \widehat{T}(i-2), i \geq 1\right\} .
$$

Lemma 2. For each $d \in M$ there exists $s \geq 1$ such that $d^{p^{s}} \in \mathcal{D}_{2}$.

Proof. We follow the idea from [ᄑ]. Let $\lambda=(1+\sqrt{1+4(p-1)}) / 2$, the positive root of $\lambda^{2}-\lambda-(p-1)=0$. We will define a $\mathbb{Z}+\mathbb{Z} \lambda$-grading on the algebra $\mathcal{D}$ by setting $\operatorname{deg}\left(t_{i}\right)=-\lambda^{i}$ and $\operatorname{deg}\left(\partial_{i}\right)=\lambda^{i}$. The degree of an arbitrary element from $\widehat{T}(i-2) \partial_{i}$ is greater than or equal to

$$
\begin{aligned}
\lambda^{i}-(p-1)\left(1+\cdots+\lambda^{i-2}\right) & =\lambda^{i}-(p-1) \frac{\lambda^{i-1}-1}{\lambda-1} \\
& =\frac{\lambda^{i-1}\left(\lambda^{2}-\lambda-(p-1)\right)+p-1}{\lambda-1}=\frac{p-1}{\lambda-1}=\epsilon>0 .
\end{aligned}
$$

Hence every homogeneous component of an element $d$ from $\mathcal{D}$ has degree $\geq \epsilon$ and every homogeneous component of $d^{p^{s}}$ has degree $\geq p^{s} \epsilon$.

Now let $d \in M_{k}$. Then by Lemma 1(b), $d^{p^{s}} \in M_{k+s}$, hence

$$
d^{p^{s}} \in \sum_{i=1}^{k+s} \widehat{T} \partial_{i}+T^{p} \partial_{k+s+1}+T^{2 p-1} \partial_{k+s+2}+\cdots
$$

The degree of an arbitrary nonzero element from $\sum_{i=1}^{k+s} \widehat{T} \partial_{i}$ is $\leq \lambda^{k+s}$. Hence, if the $\sum_{i=1}^{k+s} \widehat{T} \partial_{i}$-part of $d^{p^{s}}$ is not equal to 0 , then $d^{p^{s}}$ has a nonzero homogeneous component of degree $\leq \lambda^{k+s}$. Hence $p^{s} \epsilon \leq \lambda^{k+s}$. However, since $\lambda<p$ it follows that $p^{s} \epsilon>\lambda^{k+s}$ for sufficiently large $s$, a contradiction. Hence for sufficiently large $s$ we have $d^{p^{s}} \in$ $T^{p} \partial_{k+s+1}+T^{2 p-1} \partial_{k+s+2}+\cdots \subseteq \mathcal{D}_{2}$. 
Define

$$
T^{s} \mathcal{D}=\left\{\sum_{i=1}^{r} a_{i} d_{i} \mid a_{i} \in T^{s}, d_{i} \in \mathcal{D}, r \geq 1\right\} .
$$

Since $\bigcap_{s \geq 1} T^{s} \mathcal{D}=(0)$ the subspaces $T^{s} \mathcal{D} \cap \mathcal{D}$ define a topology on $\mathcal{D}$. Let

$$
\mathcal{D}_{\text {fin }}=\left\{\sum_{i=1}^{r} a_{i} \partial_{i} \mid a_{i} \in \widehat{T}(i-2), r \geq 1\right\}
$$

and consider the closure of $\mathcal{D}_{\text {fin }}$ in the above topology: $\overline{\mathcal{D}}_{\text {fin }}=\bigcap_{s \geq 1}\left(\mathcal{D}_{\text {fin }}+\left(T^{s} \mathcal{D} \cap \mathcal{D}\right)\right)$.

Lemma 3. $\overline{\mathcal{D}}_{\text {fin }}$ is a subalgebra of $\mathcal{D}$, closed with respect to $p$-th powers.

Proof. We have $\left[\mathcal{D}, T^{s} \mathcal{D}\right] \subseteq T^{s-1} \mathcal{D}$. This implies

$$
\left[\mathcal{D}_{\text {fin }}+\left(T^{k} \mathcal{D} \cap \mathcal{D}\right), \mathcal{D}_{\text {fin }}+\left(T^{s} \mathcal{D} \cap \mathcal{D}\right)\right] \subseteq \mathcal{D}_{\text {fin }}+\left(T^{\min (k, s)-1} \mathcal{D} \cap \mathcal{D}\right)
$$

Hence, $\left[\overline{\mathcal{D}}_{\text {fin }}, \overline{\mathcal{D}}_{\text {fin }}\right] \subseteq \overline{\mathcal{D}}_{\text {fin }}$.

Let $a \in \mathcal{D}_{\text {fin }}+\left(T^{s} \mathcal{D} \cap \mathcal{D}\right)$. Thus there exist elements $c_{1}, \ldots, c_{r} \in T^{s}$ and an integer $k \geq 1$ such that $a=\sum_{i=1}^{\infty} a_{i} \partial_{i}$ and all the coefficients $a_{k}, a_{k+1}, \ldots$ lie in $\sum_{j=1}^{r} c_{j} \widehat{T}$. As above we notice that $\left(a_{i} \partial_{i}\right)^{p}=0$ and $\left[a_{i_{1}} \partial_{i_{1}}, \ldots, a_{i_{p}} \partial_{i_{p}}\right]=b \partial_{i_{q}}$, where the coefficient $b$ is an expression in derivatives of $a_{i_{1}}, \ldots, a_{i_{p}}$ and $i_{q}=\max \left\{i_{1}, \ldots, i_{p}\right\}$. Suppose that $i_{q} \geq k$. Then $a_{i_{q}} \in \sum c_{j} \widehat{T}$ and $b$ lies in the ideal of $\widehat{T}$ generated by the elements $\partial_{j_{1}} \cdots \partial_{j_{l}} c_{j}$, where $l \leq p-1$. This implies that $a^{p} \in \mathcal{D}_{\text {fin }}+\left(T^{s-p+1} \mathcal{D} \cap \mathcal{D}\right)$.

Now let

$$
L=M \cap \overline{\mathcal{D}}_{\text {fin }}
$$

Lemma 4. For each $a \in L$ there exists $s \geq 1$ such that $a^{p^{s}} \in T^{2} \mathcal{D}$.

Proof. By Lemma 2 there exists $s \geq 1$ such that $a^{p^{s}} \in \mathcal{D}_{2}$. On the other hand, $a^{p^{s}} \in$ $\mathcal{D}_{\text {fin }}+\left(T^{2} \mathcal{D} \cap \mathcal{D}\right)$. We claim that $\mathcal{D}_{2} \cap\left(\mathcal{D}_{\text {fin }}+T^{2} \mathcal{D}\right) \subseteq T^{2} \mathcal{D}$. Indeed, let $d \in \mathcal{D}_{2}, d=$ $d^{\prime}+d^{\prime \prime}, d^{\prime} \in \mathcal{D}_{\text {fin }}, d^{\prime \prime} \in T^{2} \mathcal{D}$. Then $d^{\prime}=d-d^{\prime \prime} \in \mathcal{D}_{\text {fin }} \cap \mathcal{D}_{2} \subseteq T^{2} \mathcal{D}$.

Lemma 5. The associative subalgebra of $\operatorname{End}_{F}(\widehat{T})$ generated by $T^{2} \mathcal{D}$ is locally nilpotent.

Proof. Consider a collection of elements $a_{i}^{\prime} a_{i}^{\prime \prime} d_{i}$, where $1 \leq i \leq r, a_{i}^{\prime}, a_{i}^{\prime \prime} \in T$, and $d_{i} \in \mathcal{D}$. Let $A$ be the subalgebra of $T$ generated by $a_{i}^{\prime}, a_{i}^{\prime \prime}, 1 \leq i \leq r$. Since $a_{i}^{\prime p}=a_{i}^{\prime \prime p}=0$ it follows that $A^{2 r(p-1)+1}=(0)$. Now, $\left(a_{i_{1}}^{\prime} a_{i_{1}}^{\prime \prime} d_{i_{1}}\right) \cdots\left(a_{i_{s}}^{\prime} a_{i_{s}}^{\prime \prime} d_{i_{s}}\right)=\sum b_{1} \cdots b_{2 s} d_{j_{1}} \cdots d_{j_{q}}$, where $q \leq s$ and the $b_{i}$ 's are obtained from $a_{j}^{\prime}, a_{j}^{\prime \prime}, 1 \leq j \leq r$, via (repeated) applications of the derivations $d_{k}$. Since there are only $s$ derivations $d_{i_{1}}, \ldots, d_{i_{s}}$ it follows that at least $s$ of $b_{1}, \ldots, b_{2 s}$ lie in $\left\{a_{i_{1}}^{\prime}, a_{i_{2}}^{\prime \prime}, \ldots, a_{i_{s}}^{\prime}, a_{i_{s}}^{\prime \prime}\right\}$. If $s=2 r(p-1)+1$, then $\left(a_{i_{1}}^{\prime} a_{i_{1}}^{\prime \prime} d_{i_{1}}\right) \cdots\left(a_{i_{s}}^{\prime} a_{i_{s}}^{\prime \prime} d_{i_{s}}\right)=0$.

Lemmas 4 and 5 imply 
Proposition 1. All elements of L are nilpotent.

Simliarly to $[\overline{\mathrm{P}}]$ we can find finitely generated nonnilpotent subalgebras in $L$ and estimate their Gelfand-Kirillov dimensions.

Example 1. Consider the elements

$$
v_{n}=\partial_{n}+\sum_{i=n+1}^{\infty}\left(t_{n-1} \cdots t_{i-2}\right)^{p-1} \partial_{i}
$$

from $L, n \geq 1$. We have

$$
\begin{gathered}
{\left[v_{i}, v_{i+1}\right]=-t_{i}^{p-2} v_{i+2} ; \quad\left[v_{i}, v_{j}\right]=-\left(t_{i-1} \cdots t_{j-3}\right)^{p-1} t_{j-1}^{p-2} v_{j+1} \quad \text { for } j \geq i+2} \\
v_{i}\left(t_{j}\right)= \begin{cases}0, & j<i \\
1, & j=i \\
\left(t_{i-1} \cdots t_{j-2}\right)^{p-1} & j>i .\end{cases}
\end{gathered}
$$

It is easy to see that the Lie algebra $\mathcal{L}=\operatorname{Lie}\left\langle v_{1}, v_{2}\right\rangle$ generated by $v_{1}, v_{2}$ is not nilpotent. Just as in $[\overline{\mathrm{P}}]$, we will find its Gelfand-Kirillov dimension.

Proposition 2. $\operatorname{GKdim} \mathcal{L}=\log _{\lambda} p$.

Clearly, $1<\log _{\lambda} p<2$ and $\log _{\lambda} p \rightarrow 2$ as $p \rightarrow \infty$.

We first prove several lemmas.

Lemma 6. We have

$$
\mathcal{L} \subseteq \operatorname{Span}\left(t_{0}^{i_{0}} \cdots t_{n-2}^{i_{n-2}} v_{n} \mid n \geq 1 ; 0 \leq i_{0}, \ldots, i_{n-4} \leq p-1 ; 0 \leq i_{n-3}, i_{n-2} \leq p-2\right) .
$$

Proof. Denote the right hand side of this inclusion by $V$. Since all $v_{i}$ are in $V$, it suffices to prove that $\left[V, v_{1}\right],\left[V, v_{2}\right] \subseteq V$. Let $a=t_{0}^{i_{0}} t_{1}^{i_{1}} \cdots t_{n-2}^{i_{n-2}}$, $a v_{n} \in V$. Consider

$$
\begin{aligned}
{\left[a v_{n}, v_{1}\right] } & =a\left[v_{n}, v_{1}\right]-v_{1}(a) v_{n} \\
& =-a\left(t_{0} \cdots t_{n-3}\right)^{p-1} t_{n-1}^{p-2} v_{n+1}-\sum_{s=0}^{n-2} t_{0}^{i_{0}} \cdots t_{s-1}^{i_{s-1}} v_{1}\left(t_{s}^{i_{s}}\right) t_{s+1}^{i_{s+1}} \cdots t_{n-2}^{i_{n-2}} v_{n}
\end{aligned}
$$

The first term clearly lies in $V$ and so do all the summands in the second term for $s \leq n-3$. For $s=n-2$ we have

$$
t_{0}^{i_{0}} \cdots t_{n-3}^{i_{n-3}} v_{1}\left(t_{n-2}^{i_{n-2}}\right) v_{n}=i_{n-2} t_{0}^{i_{0}} \cdots t_{n-3}^{i_{n-3}}\left(t_{0} \cdots t_{n-4}\right)^{p-1} t_{n-2}^{i_{n-2}-1} v_{n} \in V .
$$

Similarly, $\left[a v_{n}, v_{2}\right] \in V$. 
Observe that the Lie algebra $\mathcal{L}$ is a graded subalgebra of the $\mathbb{Z}+\mathbb{Z} \lambda$-graded algebra $\mathcal{D}$ with $\operatorname{deg}\left(v_{1}\right)=\lambda, \operatorname{deg}\left(v_{2}\right)=\lambda^{2}$. Let $\mathcal{L}_{d}$ be the sum of the homogeneous subspaces of $\mathcal{L}$ of degree $\leq d$.

Suppose that $v=t_{0}^{i_{0}} \cdots t_{n-4}^{i_{n-4}} t_{n-3}^{i_{n-3}} t_{n-2}^{i_{n-2}} v_{n} \in \mathcal{L}_{d}$ with $0 \leq i_{0}, \ldots, i_{n-4} \leq p-1$ and $0 \leq i_{n-3}, i_{n-2} \leq p-2$. Then

$$
\begin{aligned}
\lambda^{n}-(p-1)(1+\lambda+\cdots+ & \left.\lambda^{n-4}\right)-(p-2)\left(\lambda^{n-3}+\lambda^{n-2}\right) \\
& =\lambda^{n}-(p-1)\left(1+\lambda+\cdots+\lambda^{n-2}\right)+\lambda^{n-3}+\lambda^{n-2} \\
& =\frac{p-1}{\lambda-1}+\lambda^{n-3}+\lambda^{n-2} \leq \operatorname{deg}(v) \leq d .
\end{aligned}
$$

This implies that $2 \lambda^{n-3} \leq d$ and therefore $n \leq \log _{\lambda}(d / 2)+3$.

Now,

$$
\operatorname{dim} \mathcal{L}_{d} \leq \sum_{n \leq \log _{\lambda}(d / 2)+3} p^{n-3} \leq p^{\log _{\lambda}(d / 2)+3} \leq c d^{\log _{\lambda} p},
$$

where $c$ is a constant which depends on $p$.

Now let us estimate the dimension of $\mathcal{L}_{d}$ from below. In what follows we will assume that $p \geq 3$. For $p=2$ the assertion was proved in [ [PS].

Lemma 7. For each $n \geq 1$ there exists $a_{2 n-3} \in \widehat{T}(2 n-3)$ such that

$$
v_{2 n} \in \mathcal{L}, \quad t_{2 n-1}^{p-2} v_{2 n+1} \in \mathcal{L}, \quad v_{2 n-1}^{\prime}=v_{2 n-1}+a_{2 n-3} t_{2 n-2}^{p-2} v_{2 n} \in \mathcal{L} .
$$

Proof. For $n=1$ the assertion is obvious. Let us assume it for $n$ and prove for $n+1$. We have

$$
t_{2 n-1}^{p-2} t_{2 n}^{p-2} v_{2 n+2}=\left[t_{2 n-1}^{p-2} v_{2 n+1}, v_{2 n}\right] \in \mathcal{L}
$$

Since $\left[v_{2 n+2}, v_{2 n}\right]=t_{2 n-1}^{p-1} t_{2 n+1}^{p-2} v_{2 n+3}$ and since $p \geq 3$, it follows that

$$
\left[t_{2 n-1}^{p-2} t_{2 n}^{p-2} v_{2 n+2}, v_{2 n}\right]=(p-2) t_{2 n-1}^{p-2} t_{2 n}^{p-3} v_{2 n+2}
$$

Repeating this $p-2$ times we get

$$
(p-2) ! t_{2 n-1}^{p-2} v_{2 n+2} \in \mathcal{L} .
$$

For $i>0$ we have $\left[t_{2 n-1}^{i} v_{2 n+2}, v_{2 n}\right]=0$. Hence

$$
\left[t_{2 n-1}^{i} v_{2 n+2}, v_{2 n-1}^{\prime}\right]=\left[t_{2 n-1}^{i} v_{2 n+2}, v_{2 n-1}\right] .
$$

Commuting $p-2$ times, we get

$$
\begin{aligned}
{\left[\ldots[t_{2 n-1}^{p-2} v_{2 n+2}, \underbrace{\left.v_{2 n-1}^{\prime}\right], \ldots, v_{2 n-1}^{\prime}}_{p-2}]\right.} & =\left[\ldots\left[t_{2 n-1}^{p-2} v_{2 n+2}, v_{2 n-1}\right], \ldots, \ldots, v_{2 n-1}\right] \\
& =(p-2) ! v_{2 n+2} \in \mathcal{L},
\end{aligned}
$$

which proves the first inclusion of the lemma. 
Now,

$$
\left[v_{2 n+2}, t_{2 n-1}^{p-2} v_{2 n+1}\right]=t_{2 n-1}^{p-2} t_{2 n+1}^{p-2} v_{2 n+3} \in \mathcal{L} .
$$

As above, for any $i>0$ we have

$$
\left[t_{2 n-1}^{i} t_{2 n+1}^{p-2} v_{2 n+3}, v_{2 n-1}^{\prime}\right]=\left[t_{2 n-1}^{i} t_{2 n+1}^{p-2} v_{2 n+3}, v_{2 n-1}\right]
$$

Hence,

$$
\begin{aligned}
{\left[\ldots[t_{2 n-1}^{p-2} t_{2 n+1}^{p-2} v_{2 n+3}, \underbrace{\left.v_{2 n-1}^{\prime}\right], \ldots, v_{2 n-1}^{\prime}}_{p-2}]\right.} & =\left[\ldots[t_{2 n-1}^{p-2} t_{2 n+1}^{p-2} v_{2 n+3}, \underbrace{\left.v_{2 n-1}\right], \ldots, v_{2 n-1}}_{p-2}]\right. \\
& =(p-2) ! t_{2 n+1}^{p-2} v_{2 n+3},
\end{aligned}
$$

which proves the second inclusion of the lemma.

Finally,

$$
\begin{aligned}
& \mathcal{L} \ni\left[t_{2 n-1}^{p-2}\right.\left.v_{2 n+1}, v_{2 n-1}^{\prime}\right] \\
& \quad=\left[t_{2 n-1}^{p-2} v_{2 n+1}, v_{2 n-1}\right]+\left[t_{2 n-1}^{p-2} v_{2 n+1}, a_{2 n-3} t_{2 n-2}^{p-2} v_{2 n}\right] \\
& \quad=(p-2) t_{2 n-1}^{p-3} v_{2 n+1}+t_{2 n-1}^{p-2} t_{2 n}^{p-2} v_{2 n+2}+a_{2 n-3} t_{2 n-2}^{p-2} t_{2 n-1}^{p-2} t_{2 n}^{p-2} v_{2 n+2}
\end{aligned}
$$

Write

$$
A_{i}=t_{2 n-1}^{i} v_{2 n+1}, \quad B_{i}=t_{2 n-1}^{i} t_{2 n}^{p-2} v_{2 n+2}, \quad C_{i}=a_{2 n-3} t_{2 n-2}^{p-2} t_{2 n-1}^{i} t_{2 n}^{p-2} v_{2 n+2} .
$$

For any $i>0$ we have

$$
\begin{aligned}
& {\left[A_{i}, v_{2 n-1}^{\prime}\right]=\left[A_{i}, v_{2 n-1}\right]+\left[A_{i}, a_{2 n-3} t_{2 n-2}^{p-2} v_{n}\right]=i A_{i-1}+B_{i}+C_{i},} \\
& {\left[B_{i}, v_{2 n-1}^{\prime}\right]=\left[B, v_{2 n-1}\right]=i B_{i-1},} \\
& {\left[C_{i}, v_{2 n-1}^{\prime}\right]=\left[C_{i}, v_{2 n-1}\right]=i C_{i-1} .}
\end{aligned}
$$

Therefore, for all $0 \leq i \leq p-2$ we have

$$
\begin{aligned}
& \mathcal{L} \ni\left[\ldots[A_{p-2}, \underbrace{\left.v_{2 n-1}^{\prime}\right], \ldots, v_{2 n-1}^{\prime}}_{i}]\right. \\
&=\frac{(p-2) !}{(p-i-1) !}\left((p-i-1) A_{p-i-2}+i B_{p-i-1}+i C_{p-i-1}\right) .
\end{aligned}
$$

In particular, for $i=p-2$ we get

$$
\mathcal{L} \ni A_{0}+(p-2) B_{1}+(p-2) C_{1}=v_{2 n+1}+a_{2 n-1} t_{2 n}^{p-2} v_{2 n+2},
$$

where $a_{2 n-1}=-2\left(t_{2 n-2}+a_{2 n-3}\right) t_{2 n-2}^{p-2} t_{2 n-1} \in T(2 n-1)$. 
Lemma 8. The algebra $\mathcal{L}$ contains all elements of the type

$$
t_{0}^{p-1} t_{1}^{i_{1}} \cdots t_{2 n-3}^{i_{2 n-3}} t_{2 n-1}^{p-2} v_{2 n+1}
$$

with $0 \leq i_{k} \leq p-1$ for $k=1, \ldots, 2 n-3$ and $n \geq 2$.

Proof. Consider in $\mathcal{L}$ the element $l=\left[v_{2 n}, v_{1}\right]=\left(t_{0} \cdots t_{2 n-3}\right)^{p-1} t_{2 n-1}^{p-2} v_{2 n+1}$. Note that

$$
\left[l, v_{2 n-3}^{\prime}\right]=\left[l, v_{2 n-3}\right]=(p-1)\left(t_{0} \cdots t_{2 n-2}\right)^{p-1} t_{2 n-3}^{p-2} t_{2 n-1}^{p-2} v_{2 n+1} \in \mathcal{L} .
$$

Continuing in this way, we find that $\left(t_{0} \cdots t_{2 n-2}\right)^{p-1} t_{2 n-3}^{i} t_{2 n-1}^{p-2} v_{2 n+1} \in \mathcal{L}$ for all $i=$ $p-1, \ldots, 0$.

Assume that, for some $1 \leq k \leq 2 n-3, \mathcal{L}$ contains all elements of the type

$$
l_{k}=\left(t_{0} \cdots t_{k}\right)^{p-1} t_{k+1}^{i_{k+1}} \cdots t_{2 n-3}^{i_{2 n-3}} t_{2 n-1}^{p-2} v_{2 n+1}, \quad 0 \leq i_{s} \leq p-1, s=k+1, \ldots, 2 n-3 .
$$

If $k$ is even then $v_{k} \in \mathcal{L}$, and we obtain elements in $\mathcal{L}$ of type $l_{k-1}$ by commuting $l_{k}$ with $v_{k}$. If $k$ is odd then $v_{k}^{\prime} \in \mathcal{L}$, and it is easy to see that $\left[l_{k}, v_{k}^{\prime}\right]=\left[l_{k}, v_{k}\right]$, which again gives all elements of type $l_{k-1}$. Downward induction on $k$ proves that the elements of type $l_{0}$ lie in $\mathcal{L}$, which are exactly the elements $(*)$.

End of proof of Proposition 2. Let $v=t_{0}^{p-1} t_{1}^{i_{1}} t_{2}^{i_{2}} \cdots t_{2 n-3}^{i_{2 n-3}} t_{2 n-1}^{p-2} v_{2 n+1}$ where $0 \leq i_{k} \leq$ $p-1$ for $k=1, \ldots, 2 n-3$ and $n \geq 2$. Then $\operatorname{deg}(v)<\operatorname{deg}\left(v_{2 n+1}\right)=\lambda^{2 n+1}$. If $n \leq \frac{1}{2}\left(\log _{\lambda} d-1\right)$, then $\lambda^{2 n+1} \leq d$ and therefore $v \in \mathcal{L}_{d}$. This implies

$$
\operatorname{dim} \mathcal{L}_{d} \geq \sum_{2 \leq n \leq \frac{1}{2}\left(\log _{\lambda} d-1\right)} p^{2 n-3} \geq p^{\log _{\lambda} d-4}=\frac{1}{p^{4}} d^{\log _{\lambda} p} .
$$

Summarizing we get

$$
\frac{1}{p^{4}} d^{\log _{\lambda} p} \leq \operatorname{dim} \mathcal{L}_{d} \leq c d^{\log _{\lambda} p}
$$

This implies

$$
\lim _{d \rightarrow \infty} \frac{\ln \operatorname{dim} \mathcal{L}_{d}}{\ln d}=\log _{\lambda} p .
$$

Let $\mathcal{L}_{(d)}$ denote the span of all commutators in $v_{1}, v_{2}$ of length $\leq d$. It is easy to see that

$$
\mathcal{L}_{\lambda d} \subseteq \mathcal{L}_{(d)} \subseteq \mathcal{L}_{\lambda^{2} d}
$$

Hence

$$
\operatorname{GKdim} \mathcal{L}=\lim _{d \rightarrow \infty} \frac{\ln \operatorname{dim} \mathcal{L}_{(d)}}{\ln d}=\lim _{d \rightarrow \infty} \frac{\ln \operatorname{dim} \mathcal{L}_{d}}{\ln d}=\log _{\lambda} p .
$$

Let $A$ be the associative subalgebra of $\operatorname{End}_{F}(\widehat{T})$ generated by $\mathcal{L}$.

Proposition 3. $\operatorname{GKdim} A \leq 2 \log _{\lambda} p$. 
Proof. The proof is similar to that in [PS]. That is why we will only sketch it. First, notice that $A$ is contained in the span of operators of the type

$$
a=t_{0}^{\alpha_{0}} \cdots t_{n-2}^{\alpha_{n-2}} v_{1}^{\beta_{1}} \cdots v_{n}^{\beta_{n}},
$$

where $0 \leq \alpha_{0}, \ldots, \alpha_{n-3} \leq p-1,0 \leq \alpha_{n-2} \leq p-2,0 \leq \beta_{1}, \ldots, \beta_{n} \leq p-1, \beta_{n} \geq 1$. Let $a \in A_{d}$. Then

$$
\begin{aligned}
d & \geq \operatorname{deg}(a)=\sum_{i=1}^{n} \beta_{i} \lambda^{i}-\sum_{j=0}^{n-2} \alpha_{j} \lambda^{j} \geq \lambda^{n}-(p-1) \sum_{j=0}^{n-3} \lambda^{j}-(p-2) \lambda^{n-2} \\
& =\frac{p-1}{\lambda-1}+\lambda^{n-2}>\lambda^{n-2}
\end{aligned}
$$

Hence, $n<\log _{\lambda} d+2=r$. For each $n$ the number of such monomials is less than $p^{2 n-1}$. Hence,

$$
\operatorname{dim} A_{d} \leq \sum_{n<r} p^{2 n-1}<p^{2 r-1}=p^{2 \log _{\lambda} d+3}
$$

Now it remains to notice that

$$
\lim _{d \rightarrow \infty} \frac{\ln p^{2 \log _{\lambda} d+3}}{\ln d}=2 \log _{\lambda} p .
$$

Example 2. The Lie algebra $L_{m}, m \geq 1$, generated by the derivations $\partial_{1}, \ldots, \partial_{m}$, $\partial_{m+1}+\sum_{i=2}^{\infty}\left(t_{1} \cdots t_{i-1}\right)^{p-1} \partial_{m+i}$ is not nilpotent. The associative subalgebra of $\operatorname{End}_{F}(\widehat{T})$ generated by $L_{m}$ has finite Gelfand-Kirillov dimension.

Acknowledgments. The authors are grateful to V. Petrogradsky and L. Small for helpful discussions.

Research of I. P. Shestakov was partially supported by the CNPq grant 304991/2006-6 and the FAPESP grants 05/60337-2, 05/60142-7.

Research of E. Zelmanov was partially supported by the NSF grants FRG DMS-0455906, DMS-0500568 and the CNPq grant 454141/2005-0.

\section{References}

[B] Bartholdi, L.: Branch rings, thinned rings, tree enveloping rings. Israel J. Math. 154, 93-139 (2006) Zbl pre05239667 MR 2254535

[BG] Bartholdi, L., Grigorchuk, R. I.: Lie Methods in Growth of Groups and Groups of Finite Width. London Math. Soc. Lecture Note Ser. 275, Cambridge Univ. Press, Cambridge (2000) Zbl 1032.20026 MR 1776763

[GS] Golod, E. S.: On nilalgebras and finitely approximable $p$-groups. Izv. Akad. Nauk SSSR 28, 273-276 (1964) Zbl 0215.39202 MR 0161878

[LS] Lenagan, T. H., Smoktunowicz, A.: An infinite dimensional affine nil algebra with finite Gelfand-Kirillov dimension. J. Amer. Math. Soc. 20, 989-1001 (2007) Zbl pre05177851 MR 2328713

[P] Petrogradsky, V. M.: Examples of self-iterating Lie algebras. J. Algebra 302, 881-886 (2006) Zbl 1109.17008 MR 2293788

[PS] Petrogradsky, V. M., Shestakov, I. P.: Examples of self-iterating Lie algebras 2. J. Lie Theory, to appear 